\title{
Marmosets that miss social cues may mimic autism
}

\section{BY KATE YANDELL}

29 JULY 2015

Ryan Ladbrook/Shutterstock.com Prime primates: Marmosets are social animals, making them an attractive option for studying features of autism.

Marmosets exposed to an epilepsy drugin the womb lack the innate ability to recognize reciprocity - the social give-and-take that is a challenge for some people with autism. The findings, published29 June inBehavioural Brain Research, add to mounting evidence that marmosets offer clues about autism¹.

The past few years have brought a surge of interest in marmoset models of autism. The tiny monkeysare highly social, living in family units that collectively care for the youngest members.Some researchers are studying marmosets missing MeCP2, the Rett syndrome gene, and others are targeting additional autism-related genes in the monkeys.

But to validate these marmoset models, researchers need to evaluate the animals' social behavior.A previous study demonstrated that marmosets are more likely to accept food from people whom they have seen share food with others than from people who refuse to share ${ }^{2}$.

In the new study, the same research team gave this social intuition test to marmosets exposed in the womb to valproic acid (VPA).Prenatal exposure to this epilepsy drug increases the risk of autism in people. 


\section{Spectrum | Autism Research News}

https://www.spectrumnews.org

The researchers enlisted actors to perform short playsfor four marmosets exposed to VPA and four untreated controls. In one play, a pair of actors exchanged pieces of steamed buns and potatoes. In a second play, one actor took food from his or her partner but gave nothing in return. The actors performed each of the two plays 108 times per marmoset.

After each skit, both actors offered a piece of sponge cake to the marmosets. Untreated marmosets accepted food readily from both actors in the sharing scenario. But when one actor hoarded the food, the monkeys chose to accept food primarily from the actor's partner. This suggests that marmosets recognize reciprocity and trust the generous actor more.

By contrast, the marmosets exposed to VPA did not discriminate between the actors in the hoarding scenario. The monkeys were as likely to accept food from the stingy actor as from the generous one.

The results suggest that the VPA-exposed monkeys lack either the motivation to study the actors' social dynamics or the ability to understand them. Social apathy and difficulty interpreting social situations are both associated with autism.

The marmosets exposed to VPA appear to have normal intelligence. They performed well on a test that requires learning which of two covered holes contains food. So their lack of preference for a generous benefactor is unlikely to arise from, say, a basic inability to remember the events of the play.

Researchers have exposed fetal mice and rats to VPA to create models of autism. These animals show more primitive social deficits, such as indifference toward spending time with other rodents. Marmosets exposed to VPA could model deficits in more sophisticated social interactions, the researchers say.

The researchers have unpublished data on other aspects of social behavior, restricted interests and obsessive behaviors in marmosets. They are also exploring whether VPA-exposed marmosets show changes in gene expression or brain structure.

In the meantime, they say their reciprocity-recognition test may help other researchers assess social behavior in genetic marmoset models of autism.

Cite this article: https://doi.org/10.53053/PDNO5384

\section{References:}

1. Yasue M. et al. Behav. Brain Res. 292, 323-326 (2015) PubMed

2. Kawai N. et al. Biol. Lett. 10, 20140058 (2014) PubMed 